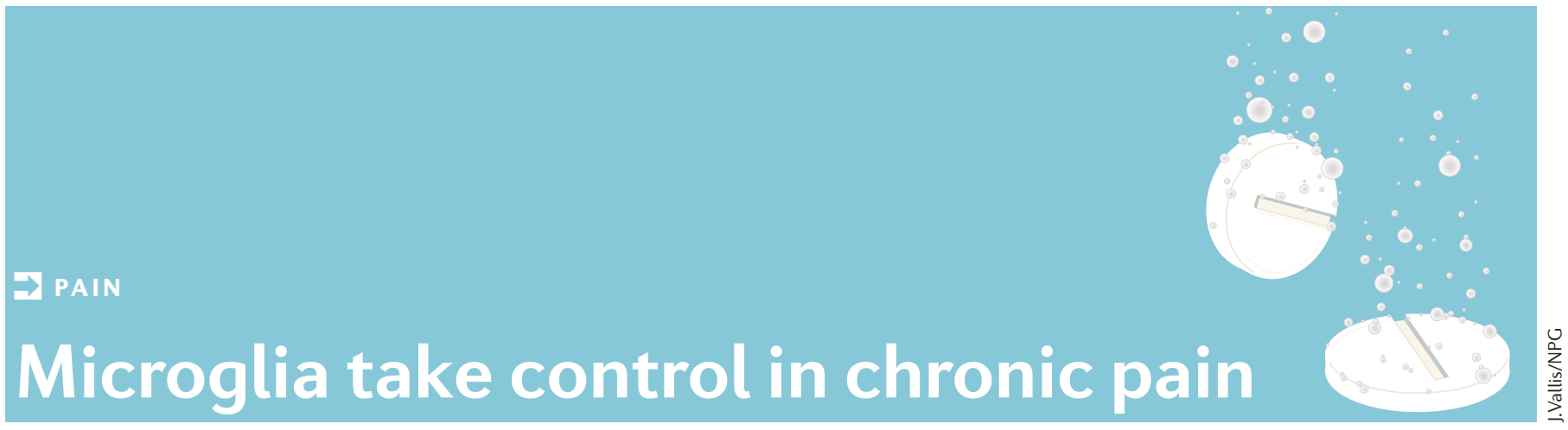

Opiates are the first-line treatment for pain, but they have two problematic side effects: tolerance and, paradoxically, hyperalgesia. The mechanism underlying hyperalgesia is poorly understood, but a new study by Ferrini et al. suggests that microglia-mediated changes in spinal $\mathrm{Cl}^{-}$homeostasis are crucial.

Opioid receptor-expressing lamina I neurons that project to the brain are key components of the pain processing pathway, and enhanced activity in this pathway has been associated with morphine-induced hyperalgesia (MIH). In addition, activity of these neurons is known to be increased following disruption of $\mathrm{Cl}^{-}$homeostasis, which is controlled by the $\mathrm{K}^{+} / \mathrm{Cl}^{-}$co-transporter $\mathrm{KCC} 2$. The authors found that a chronic morphine treatment regimen in mice that induced both tolerance and pain hypersensitivity also resulted in decreased KCC2 expression and disrupted $\mathrm{Cl}^{-}$homeostasis.

Lamina I neurons are modulated by neuronal inputs and neighbouring microglia, and previous studies have implicated P2X4 receptor-expressing microglia in the regulation of $\mathrm{Cl}^{-}$ homeostasis in these neurons; spinal microglia also express $\mu$-opioid receptors that would be activated in the presence of morphine. Ablation of spinal microglia reversed established MIH, and intrathecal injection of microglia that had been incubated with chronic morphine in vitro was sufficient to induce hyperalgesia in naive animals. Both the acquisition of MIH and its ongoing expression was prevented by pharmacological inhibition and genetic ablation of P2X 4 receptors.

It is known that microglial P2X4 receptor activation causes brain-derived neurotrophic factor (BDNF) release, which in turn results in reduced KCC2 expression. The authors therefore reasoned that the hyperalgesic effects of morphine might involve P2X4 receptor-dependent BDNF release from microglia, which could trigger a reduction in neuronal KCC2 levels and a consequent impairment of $\mathrm{Cl}^{-}$ homeostasis and hyperalgesia.

Mice in which microglial BDNF expression had been selectively inhibited failed to develop MIH (although the anti-nociceptive response to acute morphine and development of tolerance were unaffected).
Interestingly, using naloxone stereoisomers that differentially inhibit $\mu$-receptor signalling, the authors found that although the upregulation of spinal P2X4 receptors by morphine was $\mu$-receptor-dependent, BDNF release by microglia in which $\mathrm{P} 2 \mathrm{X} 4$ receptors were already upregulated was $\mu$-receptor-independent. That a $\mu$-receptor-independent pathway is necessary to complete this signalling cascade indicates that it is possible to prevent the hyperalgesia without interfering with the analgesic action of morphine (which is mediated by $\mu$-receptors).

Overall, these data suggest that an underlying mechanism for $\mathrm{MIH}$ involves BDNF release from microglia, which signals to lamina I neurons, causing decreased KCC2 expression, disrupted $\mathrm{Cl}^{-}$homeostasis and hyperalgesia. The data implicate the P2X4-BDNF-KCC2 pathway as a potential therapeutic target to prevent this highly deleterious side effect of morphine.

\section{Sian Lewis}

ORIGINAL RESEARCH PAPER Ferrini, F. et al. Morphine hyperalgesia gated through microgliamediated disruption of neuronal $\mathrm{Cl}^{-}$homeostasis. Nature Neurosci. 6 Jan 2013 (doi:10.1038/nn.3295) it is possible to prevent the hyperalgesia without interfering with the analgesic action of morphine (which is mediated by $\mu$-receptors) 